\title{
Editorial
}

\section{Charge Transfer in Ionic and Molecular Systems}

\author{
Marie-Christine Bacchus-Montabonel
}

Laboratoire de Spectrométrie Ionique et Moléculaire, UMR 5579, CNRS et Université Lyon I, 43 Bd. du 11 Novembre 1918, 69622 Villeurbanne Cedex, France

Received: 5 February 2002 / Published: 28 March 2002

Charge transfer reactions are fundamental processes present in almost all areas of physics, chemistry and biochemistry. Most of the time, these reactions are taking place in the condensed phase and solvent effects are determinant. Nevertheless, studies in the gas phase provide direct understanding of the intrinsic behaviour of the ions and are essential for the modeling of more complex mechanisms.

Investigations of charge exchange reactions in ion-atom, ion-molecule and, more recently, ion-ion collisions are of fundamental as well as of practical interest. These reactions are of great importance in a variety of research fields, such as the description of many astrophysical and terrestrial environments, controlled thermonuclear fusion or ion accelerator technology. For example, the interaction of solar wind ions with cometary gases, auroral ions with planetary atmospheres, and stellar wind ions with the interstellar medium involves the formation of excited states of collision partners with the resultant visible, UV an X-ray emission. Both description of thermal and ionization balance of astrophysical plasmas and modeling of ion transport and radiative cooling processes in the diverter region of current large Tokamak fusion plasma devices require information on the relevant electron capture processes.

Experimental methods such as single and double translational energy spectroscopy, energy gain spectroscopy, photon spectroscopy, Auger electron spectroscopy or recoil ion momentum spectroscopy combined with theoretical approaches, from the classical trajectory Monte-Carlo model, or the simple Landau-Zener model, to very accurate atomic or ab-initio molecular expansion methods within either time-dependent or time-independent collisional treatments, provide efficient tools for these studies. A large survey of these approaches is presented in this special issue, emphasizing recent results and developments of single-electron capture processes as well as multiple-electron capture reactions, essentially double-electron capture with regard to the complexity of the many-body problem involved, on ion-atom and ion-molecule collisions in the low-energy regime. 